\title{
In Vitro Equivalence Studies of Generic Metformin Hydrochloride Tablets and Propranolol Hydrochloride Tablets Under Biowaiver Conditions in Lagos State, Nigeria
}

\author{
Olubukola O. Oyetunde ${ }^{1, *}$, Fola Tayo ${ }^{1}$, Moshood O. Akinleye', \\ and Bolajoko A. Aina ${ }^{1}$ \\ ${ }^{1}$ Department of Clinical Pharmacy \& Biopharmacy, Faculty of Pharmacy, University of Lagos, Lagos, Nigeria \\ ${ }^{2}$ Department of Pharmaceutical Chemistry, Faculty of Pharmacy, University of Lagos, Lagos, Nigeria
}

\begin{abstract}
This study used biowaiver conditions to assess bioequivalence of some generic products used in Nigeria. None of the generic samples tested met biowaiver conditions; therefore, in vivo bioequivalence studies are required to ascertain therapeutic equivalence. To take advantage of the cost savings of using in vitro dissolution as a surrogate for bioequivalence studies, manufacturers of generic products need to consider factors that affect solubility and permeability of their products when formulating them.
\end{abstract}

\section{INTRODUCTION}

G eneric drugs are copies of innovator drug products with expired patents. They are promoted for use in practice because they are usually less expensive than the innovator products, thereby improving access to life-saving drugs, especially in developing countries. Regardless of price, generic drug quality should be comparable to that of the innovator product. Generic drug products can only be interchangeable with innovator products when they are pharmaceutically and therapeutically equivalent (1).

In vivo bioequivalence (BE) studies are commonly used to assess therapeutic equivalence, but these studies are often costly (2) and involve invasive procedures. The Biopharmaceutics Classification System (BCS) can be used to reduce in vivo $B E$ requirements (3). In vitro dissolution tests based on BCS are acceptable surrogates for establishing the bioequivalence of generics with the innovator products. According to FDA (4) and WHO (1), a biowaiver for in vivo studies can be obtained for BCS Class 1 drugs that are very rapidly dissolving ( $\geq 85 \%$ dissolved within 15 $\mathrm{min}$ ) or are rapidly dissolving ( $\geq 85 \%$ dissolved within 30 $\min$ ) with $f_{2} \geq 50$ in three dissolution media ranging in $\mathrm{pH}$ from 1.2 to 6.8. Propranolol hydrochloride is a BCS Class 1 drug (5). WHO also recommends biowaiver for Class 2 and 3 drugs that are very rapidly dissolving (1). Metformin hydrochloride is highly soluble with low permeability; it is therefore a BCS Class 3 drug (6) and is eligible for a biowaiver based on the WHO criteria. According to the WHO biowaiver testing procedure for Class 3 drugs (1), a biowaiver can be considered only if both the generic and the innovator products dissolve very rapidly (i.e., $85 \%$

*Corresponding author. or more dissolved within 15 min in standard dissolution media at $\mathrm{pH} 1.2,4.5$, and 6.8).

This study sought to apply the BCS biowaiver requirements to assess the equivalence of commonly interchanged generic propranolol hydrochloride tablets and metformin hydrochloride tablets with their respective innovator products.

\section{MATERIALS}

\section{Samples}

The drug samples investigated were all conventional, immediate-release, solid oral dosage forms. The innovator products, Inderal (IBP) for propranolol hydrochloride 40-mg tablets and Glucophage for metformin hydrochloride 500-mg tablets (IB), were purchased from country representatives. Two generic versions of propranolol hydrochloride 40-mg tablets (MINP manufactured locally and MIIP imported) and four of metformin hydrochloride 500-mg tablets (MIN1 and MIN2 manufactured locally, and MII1 and MII2 imported) were purchased from registered pharmaceutical wholesale drug outlets in Lagos State.

All reagents were analytical grade: potassium dihydrogen orthophosphate (Surechem), ammonium acetate (Riedelde Haën), hydrochloric acid (Riedel-de Haën), methanol (Sigma-Aldrich), and sodium hydroxide (Riedel-de Haën).

\section{Reference Standards}

Propranolol hydrochloride USP (Lot I1G348) was purchased from USP, and a secondary reference standard of metformin hydrochloride was obtained from Nigeria-German Chemical PLC.

\section{Methods}

Active content of generic and innovator brands were assessed using the British Pharmacopeia 2007 method (7). 
Table 1. Assay Results for Propranolol Hydrochloride Tablets

\begin{tabular}{cc}
\hline Product & Average Content $(\%)(\boldsymbol{n}=\mathbf{2 0})$ \\
\hline IBP & $98.7 \pm 2.65$ \\
\hline MINP & $100.2 \pm 5.67$ \\
\hline MIIP & $100.4 \pm 2.87$ \\
\hline
\end{tabular}

BP range $=92.5-107.5 \%$

Table 2. Assay Results for Metformin Hydrochloride Tablets

\begin{tabular}{cc}
\hline Sample & Average Content $(\%)(\boldsymbol{n}=\mathbf{2 0})$ \\
\hline IB & $102.2 \pm 0.27$ \\
\hline MIN1 & $95.8 \pm 0.09$ \\
\hline MIN2 & $102.7 \pm 0.27$ \\
\hline MII1 & $105.2 \pm 0.27$ \\
\hline MII2 & $108.7 \pm 0.27$ \\
\hline
\end{tabular}

BP range $=95.0-105.0 \%$

\section{Dissolution}

A volume of $900 \mathrm{~mL}$ of each of the following media was employed: $0.01 \mathrm{~N}$ hydrochloric acid ( $\mathrm{pH} 2)$, phosphate buffer ( $\mathrm{pH} 4.5)$, and phosphate buffer ( $\mathrm{pH}$ 6.8). Dissolution testing was performed using Electrolab Tablet Dissolution Rate Tester Model TDT-08L (USP Apparatus 2) at $50 \mathrm{rpm}$ for both products (8). The dissolution system met the USP performance verification test requirements. Twelve dosage units of each product (propranolol IBP, MINP, and MIIP and metformin IB, MIN1, MIN2, MII1, and MII2) were evaluated in the three media. Sample aliquots of $5 \mathrm{~mL}$ were taken manually with syringes fitted with stainless tubing to ensure reproducibility of sampling location. Samples were withdrawn at specified time intervals $(10,15,30,45$, and $60 \mathrm{~min}$ ) and replaced with $5 \mathrm{~mL}$ of appropriate medium. Withdrawn samples were filtered using $0.45-\mu \mathrm{m}$ Millipore filters. An Agilent UV-vis spectrophotometer (Model No. 8453) with ChemStation Software (Rev. A.10.01) was used to analyze dissolved drug in dissolution testing.

\section{RESULTS}

All assay results for samples of propranolol hydrochloride tablets were within $B P$ limits (Table 1). The innovator brand of metformin and two generic samples were within $B P$ limits, while MII 1 was slightly over and MII 2 was clearly over the limits (Table 2).

\section{Propranolol Hydrochloride Dissolution Profiles}

Propranolol hydrochloride tablets of both the generic and innovator products were rapidly dissolving ( $\geq 85 \%$ of the labeled amount released within $30 \mathrm{~min}$ ). Their profiles were mostly not superimposable (Figures 1, 2, 3); therefore, a profile comparison using $f_{2}$ testing was performed. Neither of the samples tested had $f_{2} \geq 50$ in all three media used (Table 3).

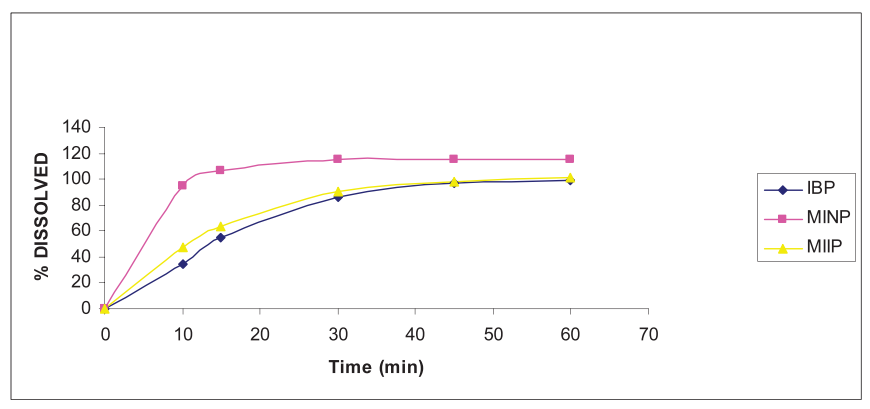

Figure 1. Dissolution rate profiles of propranolol hydrochloride tablets in $\mathrm{pH} 2$.

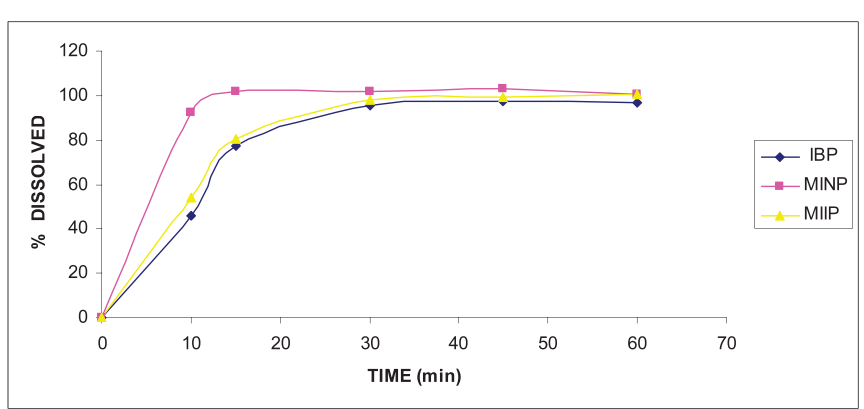

Figure 2. Dissolution rate profiles of propranolol hydrochloride tablets in $\mathrm{pH} 4.5$.

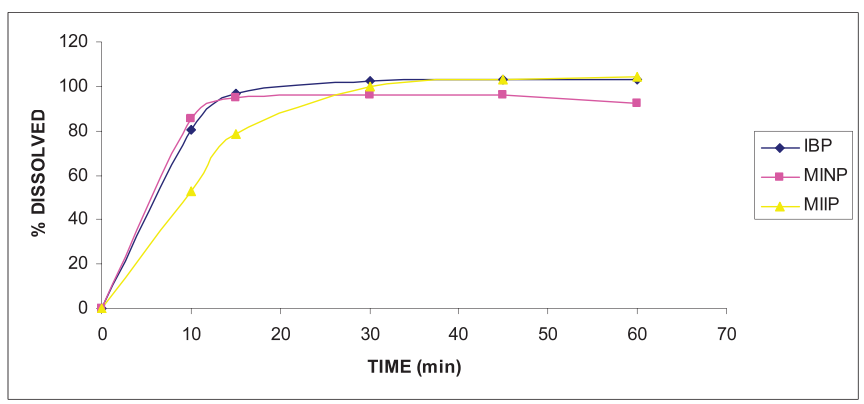

Figure 3. Dissolution rate profiles of metformin hydrochloride tablets in $\mathrm{pH} 6.8$.

\section{Metformin Dissolution Profile}

Metformin hydrochloride (BCS Class 3) test and comparator samples were not very rapidly dissolving $(\geq 85 \%$ of labeled amount not released within $15 \mathrm{~min}$ ) nor were profiles superimposable to qualify for a biowaiver according to WHO criteria (Figures 4-6 and Table 4).

\section{DISCUSSION}

Only generic drug products that are therapeutically equivalent to the innovator products and have been approved by the appropriate regulatory body may be marketed as appropriate for substitution (9). The generic drug products assessed were immediate-release oral tablets; they contained the same active ingredients as their respective reference products, propranolol $\mathrm{HCl}$ (IBP, MINP, 
Table 3. Results of $f_{2}$ Calculation for Propranolol Hydrochloride Tablets in Different pH Media

\begin{tabular}{|c|c|c|c|}
\hline & IBP (R) & MIIP (T) & MINP (T) \\
\hline Time (min) & $\%$ Dissolved & $\%$ Dissolved & \% Dissolved \\
\hline \multicolumn{4}{|l|}{ pH 2} \\
\hline 10 & 34.52 & 47.15 & 94.51 \\
\hline 15 & 54.67 & 63.02 & 106.35 \\
\hline 30 & 86.50 & 90.80 & 114.92 \\
\hline 45 & 96.96 & 98.50 & 115.44 \\
\hline \multirow[t]{2}{*}{60} & 98.84 & 101.33 & 115.58 \\
\hline & & $f_{2}=55.02$ & $f_{2}=18.79$ \\
\hline \multicolumn{4}{|l|}{ pH 4.5} \\
\hline 10 & 45.83 & 54.69 & 92.62 \\
\hline 15 & 77.38 & 80.67 & 101.51 \\
\hline 30 & 95.79 & 97.74 & 101.62 \\
\hline 45 & 97.27 & 99.31 & 102.79 \\
\hline \multirow[t]{2}{*}{60} & 96.81 & 100.55 & 100.48 \\
\hline & & $f_{2}=65.36$ & $f_{2}=31.68$ \\
\hline \multicolumn{4}{|l|}{ pH 6.8} \\
\hline 10 & 80.36 & 52.71 & 85.59 \\
\hline 15 & 96.55 & 78.46 & 94.92 \\
\hline 30 & 102.11 & 99.83 & 96.35 \\
\hline 45 & 102.85 & 102.94 & 96.25 \\
\hline \multirow[t]{2}{*}{60} & 103.20 & 104.46 & 92.57 \\
\hline & & $f_{2}=39.01$ & $f_{2}=73.97$ \\
\hline
\end{tabular}

and MIIP) and metformin $\mathrm{HCl}$ (IB, MIN1, MIN2, MII1, and MII2). They were mostly within the BP 2007 assay range for the individual active pharmaceutical ingredient assessed, with only one generic form of metformin exceeding the $B P$ upper limit.

\section{In Vitro Dissolution of Metformin}

Metformin $\mathrm{HCl}$ is a Class 3 drug according to the BCS (6). Class 3 compounds are characterized by high solubility and low permeability. For immediate-release products of this class, the assumption is that if their dissolution is very rapid under all physiological $\mathrm{pH}$ conditions, they can be expected to behave like oral solutions in vivo, since the rate-limiting step in the absorption process is permeability. Class 3 drugs are considered acceptable for biowaivers under WHO criteria (i.e., both the test and reference products are very rapidly dissolving). That is, dissolution of $85 \%$ or more of the labeled amount of API should be achieved within 15 min under all physiological conditions (1).

None of the four brands of metformin tested met this requirement because the innovator product did not achieve 85\% dissolution in 15 min (Table 4); however, a generic form (MIN1) had over 85\% dissolution within 15

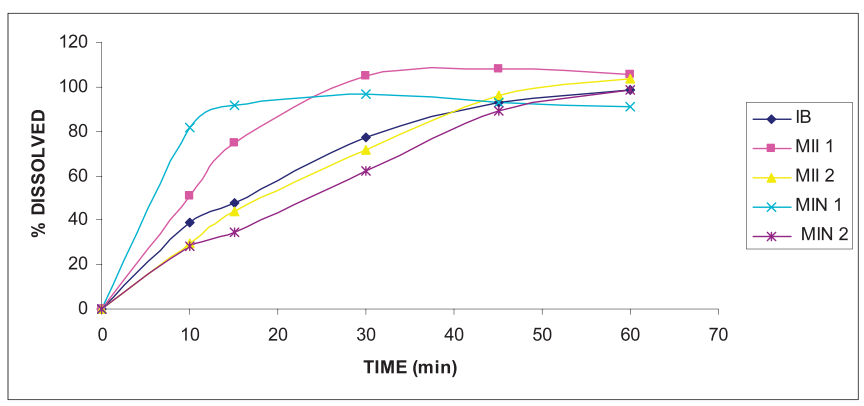

Figure 4. Dissolution rate profiles of metformin hydrochloride tablets in $\mathrm{pH} 2$.

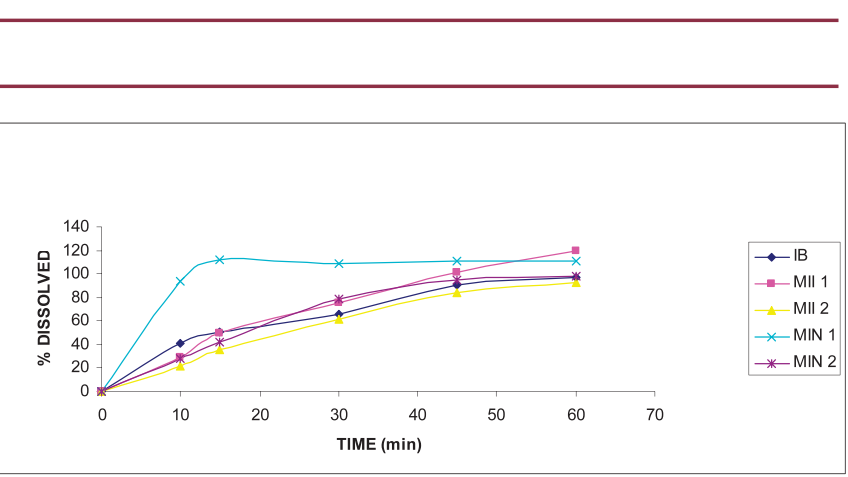

Figure 5. Dissolution rate profiles of metformin hydrochloride tablets in pH4.5.

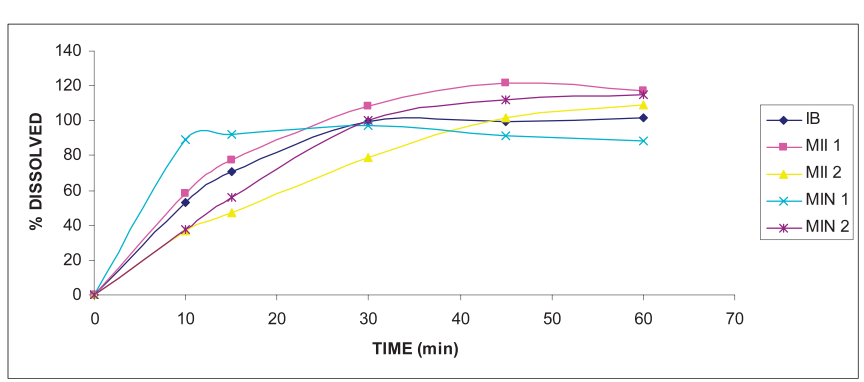

Figure 6. Dissolution rate profiles of metformin hydrochloride tablets in $\mathrm{pH} 6.8$.

min in the three media. Since MIN1 went into solution faster than the innovator product, there is the possibility of differences in the rate and extent of absorption, with the MIN1 having a higher extent of absorption. MIN2, MII1, and MII2 were not very rapidly dissolving. MII2, like IB, had fully dissolved by $45 \mathrm{~min}$, and $f_{2}$ values were greater than 50 in all physiologic $\mathrm{pH}$ conditions, showing a similarity in dissolution (Table 4). MII2 would most likely be similar to IB in rate and extent of absorption. MII2, however, exceeded the upper pharmacopeial assay limit for metformin.

According to the WHO biowaiver testing procedure, a biowaiver can be considered for BCS Class 3 drugs that are very rapidly dissolving ( $85 \%$ in $15 \mathrm{~min}$ ). None of the generic drugs tested was very rapidly dissolving. The literature, however, shows that Class 3 drugs may have similar in vivo absorption even when the dissolution is slow. In vitro-in vivo correlation has been reported in a 
Table 4. Results of $f_{2}$ Calculations for Metformin Hydrochloride Tablets in Different $p H$ Media

\begin{tabular}{|c|c|c|c|c|c|}
\hline & IB (R) & MIN 1 (T) & MIN 2 (T) & MII 1 (T) & MII 2 (T) \\
\hline Time (min) & \% Dissolved & \% Dissolved & \% Dissolved & \% Dissolved & \% Dissolved \\
\hline \multicolumn{6}{|l|}{ pH 2} \\
\hline 10 & 39.14 & 81.65 & 28.25 & 51.19 & 29.32 \\
\hline 15 & 47.60 & 91.70 & 34.29 & 74.64 & 44.03 \\
\hline 30 & 77.31 & 96.68 & 61.96 & 104.63 & 71.37 \\
\hline 45 & 92.87 & 92.99 & 89.32 & 108.23 & 96.06 \\
\hline \multirow[t]{2}{*}{60} & 98.84 & 91.23 & 98.60 & 105.42 & 103.51 \\
\hline & & $f_{2}=24.65$ & $f_{2}=46.58$ & $f_{2}=33.29$ & $f_{2}=60.03$ \\
\hline \multicolumn{6}{|l|}{ pH 4.5} \\
\hline 10 & 40.76 & 94.14 & 27.49 & 21.59 & 28.62 \\
\hline 15 & 50.44 & 112.47 & 41.78 & 35.53 & 49.03 \\
\hline 30 & 66.18 & 109.03 & 78.65 & 61.36 & 75.29 \\
\hline 45 & 90.39 & 110.54 & 94.41 & 84.08 & 100.98 \\
\hline \multirow[t]{2}{*}{60} & 97.27 & 110.79 & 97.51 & 92.59 & 119.09 \\
\hline & & $f_{2}=18.68$ & $f_{2}=51.69$ & $f_{2}=46.66$ & $f_{2}=51.49$ \\
\hline \multicolumn{6}{|l|}{ pH 6.8} \\
\hline 10 & 52.97 & 89.30 & 37.53 & 57.97 & 36.70 \\
\hline 15 & 70.60 & 91.82 & 55.75 & 77.41 & 47.12 \\
\hline 30 & 99.28 & 96.91 & 100.50 & 108.46 & 78.48 \\
\hline 45 & 99.38 & 91.67 & 112.35 & 121.78 & 101.43 \\
\hline \multirow[t]{2}{*}{60} & 101.35 & 88.45 & 114.80 & 117.17 & 109.36 \\
\hline & & $f_{2}=30.67$ & $f_{2}=45.27$ & $f_{2}=37.56$ & $f_{2}=56.91$ \\
\hline
\end{tabular}

bioequivalence study between a generic product of metformin and the innovator product, with similar dissolution profiles in three media covering the physiological $\mathrm{pH}$ range and both brands only releasing $89 \%$ within 30 min (rapidly dissolving). In a study by Cheng et al. (9), the primary bioequivalence parameters $C_{\max }, \mathrm{AUC}_{0-t, t}$ and $A \cup C_{0^{-} \infty}$ for the test products were similar to those of the reference product using log-transformed data. They concluded that a biowaiver by in vitro dissolution profiles was justified by the bioequivalence data for metformin. Blume and Schug (10) and Kortejarvi et al. (11) advocated biowaivers for Class 3 drugs that are rapidly dissolving (85\% dissolved within $30 \mathrm{~min}$ ). This advocacy was because bioavailability of this class is independent of drug dissolution; therefore, generic drugs with differing in vitro dissolution will not necessarily exhibit different in vivo performance.

The possible effect of excipients on the dissolution of metformin was not evaluated because only the innovator product Glucophage listed excipients on its packaging. Also noteworthy is the fact that even though film coating on an immediate-release product should have a negligible effect on dissolution, IB and MII2 that shared similar dissolution profiles were film-coated, whereas others were not.

\section{In Vitro Dissolution of Propranolol $\mathrm{HCl}$}

Two generic products of propranolol $\mathrm{HCl}$ (MINP and MIIP) were compared with the innovator product (IBP). According to WHO guidelines, Class 1 drugs are considered bioequivalent if in vitro dissolution shows that both the generic and innovator products are very rapidly dissolving ( $85 \%$ in $15 \mathrm{~min}$ ). If the reference (innovator) and test (generic) products are rapidly dissolving ( $85 \%$ in $30 \mathrm{~min}$ ), similarity factor calculation $\left(f_{2}\right)$ is used to assess equivalence. Similarity factor $\left(f_{2}\right)$ was used to compare the dissolution profiles in this study. There are several methods to investigate dissolution profiles comparison; the $f_{2}$ method is the simplest and is recommended by FDA (4) and WHO (1). The $f_{2}$ factor measures the closeness between the two profiles and is sensitive to large differences at any particular time point. An $f_{2}$ value of 50 or greater (50-100) ensures sameness or equivalence of the two curves and, thus, performance of the two products (12).

The $f_{2}$ calculation was applied to test the dissolution profile similarity for these products to ascertain equivalency. The $f_{2}$ values for all generic samples tested were not $\geq 50$ in all three media, suggesting that these products are not similar to the innovator product. 
The generic drugs assessed were pharmaceutically equivalent to the innovator products but were not qualified for biowaiver.

\section{CONCLUSION}

This study shows that the generic products assessed do not qualify for biowaiver; therefore, in vivo bioequivalence studies are required to ascertain BE. In vivo BE are expensive studies that, if performed, will increase the cost of drugs. Without bioequivalence studies, whether in vivo or in vitro, the therapeutic equivalence of generics is in doubt. Therefore, to use in vitro dissolution as a surrogate for bioequivalence studies for regulatory purposes, manufacturers of generic products need to consider factors that affect solubility and permeability of their products when formulating them.

\section{REFERENCES}

1. WHO Expert Committee on Specifications for Pharmaceutical Preparations. Proposal to waive in vivo bioequivalence requirements for WHO Model List of Essential Medicines immediate-release, solid oral dosage forms; WHO Technical Report Series, No. 937, Annex 7; World Health Organization: Geneva, Switzerland, 2006. http://whqlibdoc.who.int/trs/WHO_TRS_937_eng.pdf (accessed Oct 3, 2012).

2. Cook, J. A.; Bockbrader, H. N. An Industrial Implementation of the Biopharmaceutics Classification System. Dissolution Technol. 2002, 9 (2), 6-8. http:// www.dissolutiontech.com/DTresour/0502art/ DTMay02_art1.htm (accessed Oct 3, 2012).

3. Löbenberg, R; Amidon, G. L. Modern bioavailability, bioequivalence and biopharmaceutics classification system. New scientific approaches to international regulatory standards. Eur. J. Pharm. Biopharm. 2000, 50 (1), 3-12.

4. Waiver of In Vivo Bioavailability and Bioequivalence Studies for Immediate-Release Solid Oral Dosage Forms Based on a Biopharmaceutics Classification System; Guidance for Industry; U.S. Department of Health and Human Services, Food and Drug
Administration, Center for Drug Evaluation and Research (CDER), U.S. Government Printing Office: Washington, DC, 2000.

5. Volgelpoel, H.; Welink, J.; Amidon, G. L.; Junginger, H. E.; Midha, K. K.; Möller, H.; Olling, M.; Shah, V. P.; Barends, D. M. Biowaiver Monograms for Immediate Release Solid Oral Dosage Forms Based on Biopharmaceutics Classification System (BCS) Literature Data: Verapamil Hydrochloride, Propranolol Hydrochloride, and Atenolol. J. Pharm. Sci. 2004, 93 (8), 1945-1956.

6. Lindenberg, M.; Kopp, S.; Dressman, J. B. Classification of orally administered drugs on the World Health Organization Model list of Essential Medicines according to the biopharmaceutics classification system. Eur. J. Pharm. Biopharm. 2004, 58 (2), 265-278.

7. The British Pharmacopoeia 2007; The Stationary Office: London, 2007; pp 249-267.

8. Shargel, L. Drug Product Performance and Interchangeability of Multisource Drug Substances and Drug Products. Pharm. Forum 2009, 35 (3), 744-749.

9. Cheng, C. L.; Yu, L. X.; Lee, H. L.; Yang, C. Y.; Lue, C. S.; Chou, C. H. Biowaiver extension potential to BCS Class III high solubility-low permeability: bridging evidence for metformin immediate-release tablet. Eur. J. Pharm. Sci. 2004, 22 (4), 297-304.

10. Blume, H. H.; Schug, B. S. The biopharmaceutics classification system (BCS): class III drugs-better candidates for BA/BE waiver? Eur. J. Pharm. Sci. 1999, 9 (2), 117-121.

11. Kortejarvi, H.; Yliperttula, M.; Dressman, J. B.; Junginger, H. E.; Midha, K. K.; Shah, V. P.; Barends, D. M. Biowaiver Monographs for Immediate Release Solid Oral Dosage Forms: Ranitidine Hydrochloride. J. Pharm. Sci. 2005, 94 (8), 1617-1625.

12. Shah,V.; Tsong, Y.; Sathe, P.; Williams, R. Dissolution Profile Comparison Using Similarity Factor, $f_{2}$. Dissolution Technol. 1999, 6 (3), 15. http://www.dissolutiontech.com/DTresour/899Art/DissProfile.html (accessed Oct 3, 2012). 